\title{
Thermal properties of magnetic flux tubes
}

\section{Storage of flux in the solar overshoot region}

\begin{abstract}
M. Rempel ${ }^{\star}$
Max-Planck-Institut für Aeronomie, Max-Planck-Str. 2, 37191 Katlenburg-Lindau, Germany

e-mail: rempel@linmpi.mpg.de

Received 4 September 2002 / Accepted 30 October 2002

Abstract. We consider the consequences of radiative heating for the storage of magnetic flux in the overshoot region at the bottom of the solar convection zone. In the first part of the paper, we study the evolution of axisymmetric flux tubes (flux rings), which are initially in neutrally buoyant mechanical equilibrium. Radiative heating leads to a slow upward drift of the flux ring with a velocity depending on the degree of subadiabaticity of the stratification. Maintaining the flux tubes within the overshoot region for time intervals comparable with the solar cycle period requires a strongly subadiabatic stratification with $\delta=\nabla-\nabla_{\text {ad }}<-10^{-4}$, which is not predicted by most current overshoot models (e.g., Skaley \& Stix 1991; van Ballegooijen 1982; Schmitt et al. 1984). The drag force exerted by equatorward flow due to meridional circulation permits states of mechanical and thermal equilibrium in the overshoot region, but these apply only to very thin magnetic flux tubes containing less than $1 \%$ of the flux of a large sunspot. In the second part, we consider the influence of radiative heating (and cooling) on magnetic flux stored in the form of a magnetic layer. In contrast to the case of isolated flux tubes, the suppression of the convective energy transport within the magnetic layer affects the overall stratification of the overshoot region. In the case of a quenching of the convective heat conductivity by a factor of the order 100, the overshoot layer receives a net cooling leading to a stronger subadiabaticity, so that values of $\delta<-10^{-4}$ are reached. The stabilization of the stratification relaxes the conditions for flux storage. Stronger quenching of the heat conductivity leads to larger temperature perturbations (of both signs) and to the destabilization of the upper part of the overshoot layer, with the likely consequence of rapid magnetic flux loss.
\end{abstract}

Key words. MHD - Sun: magnetic fields - Sun: interior

\section{Introduction}

Solar hydromagnetic dynamo models require that large amounts of magnetic flux are retained in the convection zone for times of the order of the cycle period (Parker 1975; Galloway \& Weiss 1981; Moreno-Insertis et al. 1992). Whereas magnetic fields that do not considerably exceed the equipartition field strength with respect to the kinetic energy density of the convective motions ( $\lesssim 1 \mathrm{~T}$ ) may be transported downward by convective pumping (Tobias et al. 2001), storage of stronger magnetic field requires a mechanical equilibrium characterized by neutral buoyancy and a force balance between Coriolis force and magnetic curvature force (Moreno-Insertis et al. 1992; Rempel et al. 2000; Schüssler \& Rempel 2002). Such strong magnetic field of the order of $10 \mathrm{~T}$ at the base of solar convection zone has been inferred by various studies of the stability and rise of magnetic flux tubes (e.g., MorenoInsertis 1986; Choudhuri \& Gilman 1987; Moreno-Insertis et al. 1992; Moreno-Insertis 1992; D’Silva \& Choudhuri 1993;

^ Present address: High Altitude Observatory, National Center for Atmospheric Research, PO Box 3000, Boulder, Colorado 80307, USA, e-mail: rempel@ucar.edu
Fan et al. 1993, 1994; Schüssler et al. 1994; Caligari et al. 1995, 1998; Fisher et al. 2000).

It should be mentioned that Dorch \& Nordlund (2001) come to a different conclusion than Tobias et al. (2001) concerning the pumping of strong field. They found rescaling their results to solar values that convective pumping still works for a field strength of several Tesla.

The problem of flux storage has drawn new attention through the work of Fan \& Fisher (1996), who suggested that the equilibrium of flux tubes is disturbed by radiative heating arising from the non-vanishing divergence of the radiative heat flux in the overshoot region and deep solar convection zone. This process has been studied in detail by (Moreno-Insertis et al. 2002) in the framework of a diffusion model. Here we consider the consequences of radiative heating for the storage of magnetic flux in the overshoot region.

The paper is organized in two main parts: in Sect. 2 we discuss the influence of the radiative heating on isolated flux tubes in the overshoot region. We present numerical results and give an analytical discussion of possible equilibria. In Sect. 3 we consider the influence of radiative heating on the equilibrium of a magnetic layer as an alternative to field storage in the form 
of flux tubes. We discuss numerical and analytical solutions of the diffusion problem. Our conclusions are given in Sect. 4.

\section{Radiative heating of toroidal magnetic flux rings}

\subsection{Equations}

We consider the dynamics of strong magnetic field in the form of isolated flux tubes in the solar overshoot region, including the effects of radiative energy exchange and of transport by a meridional flow. The equations of motion are based on the approximation of thin flux tubes. Since the radiative heating is uniform along the magnetic flux tube, it favors an axisymmetric thermal instability. In order to simplify the analysis we therefore focus on $m=0$ modes in the following. For the case of axial symmetry (flux rings) considered here, the momentum equation can be written as a set of three coupled ordinary differential equations describing the (Lagrangian) motion of a mass element of the flux ring. Expressed in spherical polar coordinates, $[r(t), \theta(t), \phi(t)]$, and using a frame of reference that rotates with angular velocity $\Omega$, these equations read (Moreno-Insertis et al. 1992):

$$
\begin{aligned}
& \ddot{r}=r\left(\dot{\theta}^{2}+\sin ^{2} \theta \dot{\phi}^{2}\right)+\frac{f_{r}}{\varrho} \\
& r \ddot{\theta}=-2 \dot{r} \dot{\theta}+r \sin \theta \cos \theta \dot{\phi}^{2}+\frac{f_{\theta}}{\varrho} \\
& r \sin \theta \ddot{\phi}=-2 \dot{\phi}(\dot{r} \sin \theta+r \dot{\theta} \cos \theta)+\frac{f_{\phi}}{\varrho} .
\end{aligned}
$$

The forces $\left(f_{r}, f_{\theta}, f_{\phi}\right)$ are the sum of the magnetic curvature force, the buoyancy force, the inertial forces resulting from the rotation in the reference frame, and the drag force due to the motion of the flux ring with respect to the surrounding plasma:

$$
\begin{aligned}
f_{\mathrm{r}}= & -\frac{B^{2}}{\mu_{0} r}-\Delta \varrho\left(g-\Omega_{\mathrm{e}}^{2} r \sin ^{2} \theta\right)+2 \Omega \varrho r \sin ^{2} \theta \dot{\phi} \\
& -\varrho\left(\Omega_{\mathrm{e}}^{2}-\Omega^{2}\right) r \sin ^{2} \theta+\frac{c_{w}}{\pi R} \varrho_{\mathrm{e}}\left|\boldsymbol{v}_{\perp}\right| v_{\perp \mathrm{r}} \\
f_{\theta}= & -\frac{B^{2}}{\mu_{0} r} \cot \theta+\Delta \varrho \Omega_{\mathrm{e}}^{2} r \sin \theta \cos \theta \\
& +2 \Omega \varrho r \sin \theta \cos \theta \dot{\phi}-\varrho\left(\Omega_{\mathrm{e}}^{2}-\Omega^{2}\right) r \sin \theta \cos \theta \\
& +\frac{c_{w}}{\pi R} \varrho_{\mathrm{e}}\left|\boldsymbol{v}_{\perp}\right| v_{\perp} \theta \\
f_{\phi}= & -2 \Omega \varrho r(\dot{r} \sin \theta+r \dot{\theta} \cos \theta) .
\end{aligned}
$$

$\Delta \varrho=\varrho-\varrho_{\mathrm{e}}$ denotes the density difference between the internal and external density. $\Omega_{\mathrm{e}}$ is the angular velocity of the external plasma, $g$ the gravitational acceleration, $c_{w}$ the aerodynamic drag coefficient, and $R$ the radius of the flux tube. The perpendicular component of the velocity of the tube relative to the external plasma, $\boldsymbol{v}_{\perp}$, is given by the velocity difference between the motion of the tube and the external plasma motion (meridional circulation), $\boldsymbol{v}^{m}$ :

$$
\boldsymbol{v}_{\perp}=\left(\begin{array}{c}
v_{r}^{m}-\dot{r} \\
v_{\theta}^{m}-r \dot{\theta} \\
0
\end{array}\right)
$$

The density difference, $\Delta \varrho$, is expressed in terms of $\beta=$ $p_{\text {gas }} / p_{\text {mag }}$ and of the entropy difference, $\Delta S=S-S$ e, between the internal and the external plasma:

$$
\frac{\Delta \varrho}{\varrho}=1-\left(1+\frac{1}{\beta}\right)^{1 / \gamma} \exp \left(\frac{\Delta S}{c_{p}}\right) .
$$

The external entropy $S_{\mathrm{e}}(r)$ is given by the fixed (average) external stratification, whereas the internal entropy $S$ changes in the presence of radiative heating:

$\varrho T \dot{S}=4 \kappa \frac{T_{\mathrm{e}}-T}{R^{2}}-\operatorname{div} \boldsymbol{F}_{\mathrm{e}}$.

$T$ and $T_{\mathrm{e}}$ denote the temperatures within and outside of the flux ring, respectively. The first term on the right-hand side describes the radiative energy exchange caused by the temperature difference $\Delta T=T_{\mathrm{e}}-T$, whereas the second term represents the effect of the divergence of the radiative flux, $\boldsymbol{F}_{\mathrm{e}}$, in the exterior. This term becomes relevant in regions where the dominant mode of energy transport changes between radiative and convective (Fan \& Fisher 1996; Moreno-Insertis et al. 2002).

\subsection{The effect of radiative heating}

For the stratification of the convection zone proper we use the model of (Spruit 1977b). The detailed structure of the overshoot region is still controversial. Many models (e.g., Skaley \& Stix 1991; van Ballegooijen 1982; Schmitt et al. 1984) predict an overshoot region with a sharp transition to the underlying radiation zone, which is questioned by helioseismology (Basu et al. 1994; Christensen-Dalsgaard et al. 1995). This is not the case in the model of Xiong \& Deng (2001), which has a rather smooth transition. The thickness of the overshoot region in this model is quite large ( $\simeq 0.6$ pressure scale heights) and the subadiabaticity is considerably stronger than in non-local mixinglength models. In view of the uncertainty of overshoot models, we have decided to use a parameterized approach and consider a polytropically stratified overshoot region with a constant superadiabaticity, $\delta=\nabla-\nabla_{\text {ad }}<0$.

On the basis of Eqs. (1)-(9), we have simulated the dynamical and thermal evolution of magnetic flux rings in the overshoot region, building upon the numerical code of MorenoInsertis (1983), see also Moreno-Insertis et al. (1992). We start from a state of mechanical equilibrium, which requires neutral buoyancy and an azimuthal flow along the flux ring so that the magnetic curvature force is balanced by the Coriolis force. Choosing $\Omega$ such that the interior of the equilibrium flux ring is at rest in the rotating frame of reference, the conditions for mechanical equilibrium are (Moreno-Insertis et al. 1992):

$\Delta \varrho=0$

$\Omega=\Omega_{\mathrm{e}} \sqrt{1+\frac{B^{2}}{\mu_{0} \varrho r^{2} \sin ^{2} \theta \Omega_{\mathrm{e}}^{2}}}$.

As Moreno-Insertis et al. (1992) discussed in detail, the toroidal flow inside the flux tube can be reached by a poleward movement owing to the angular momentum conservation. 
Neutral buoyancy results from an upward movement in a subadiabatic stratification. Radiative heating disturbs the equilibrium: the resulting buoyancy causes the flux ring to drift outward. A rough estimate of the rise velocity can be obtained by assuming a quasi-stationary equilibrium between the heating and the stratification effects in the subadiabatic $(\delta<0)$ overshoot region (Fan \& Fisher 1996), viz.

$v_{r}=\frac{\nabla_{\mathrm{ad}} H_{\mathrm{p}}}{p_{\mathrm{e}}} H_{\mathrm{rad}}\left[-\delta+\left(\frac{2}{\gamma^{2}}-\frac{1}{\gamma}\right) \frac{1}{\beta}\right]^{-1}$,

where $H_{\mathrm{p}}$ indicates the pressure scale height and $H_{\mathrm{rad}}=$ $-\operatorname{div} \boldsymbol{F}_{\mathrm{e}}$. For $\delta \ll-0.12 / \beta$ (e.g., $\delta \ll-10^{-6}$ for a field strength of $10 \mathrm{~T}$ ) and using typical values for the solar overshoot region $\left(H_{\mathrm{p}}=6 \times 10^{7} \mathrm{~m}, p_{\mathrm{e}}=6 \times 10^{12} \mathrm{~Pa}\right.$, and $H_{\text {rad }}=3 \mathrm{~W} \mathrm{~m}^{-3}$ we find

$v_{r}=10\left[\frac{10^{-6}}{|\delta|}\right] \mathrm{m} \mathrm{s}^{-1}$.

We consider the rise time, $\tau$, required for an upward drift of $10^{4} \mathrm{~km}$, corresponding to the thickness of the overshoot layer, as a measure for the relevant storage time of a magnetic flux ring. Once it leaves the subadiabatically stratified overshoot region, it rapidly rises through the superadiabatically stratified convection zone proper. From the previous equation we estimate

$\tau=11\left[\frac{|\delta|}{10^{-6}}\right]$ days.

With $\delta=-3 \times 10^{-6}$ we have $\tau \approx 1$ month, which is in good agreement with the numerical results, which we now discuss.

Our restriction to the case of a constant superadiabaticity and an assumed thickness of $10^{4} \mathrm{~km}$ does not affect significantly the applicability of our results to different structures of the overshoot, as long as the relation $v_{r} \sim 1 /|\delta|$ holds, since

$\tau=\int \frac{\mathrm{d} r}{v_{r}} \sim \int|\delta| \mathrm{d} r=\langle|\delta|>\Delta r$.

By rescaling the rise time with the thickness and using the average superadiabaticity our results can be applied accordingly.

Figure 1 shows the dependence of the rise time on the initial tube radius and initial field strength. The tube radius enters the equations in two places: 1) via the drag force (per unit length), which scales with $R^{-1}$, and 2) in the first term of Eq. (9) describing the radiative inflow due to the temperature difference between flux tube and external plasma, which scales with $R^{-2}$. For decreasing tube radius, the drag force tends to increase the rise time, whereas the faster thermal exchange acts in the opposite direction. In the limit of very small radius, the latter effect is dominant, while for intermediate radii the net effect depends on the field strength as follows. In the initial neutrally buoyant equilibrium state, the temperature within the flux tube is lower than in the external plasma. Thus the heat exchange described by the first term on the right-hand side of Eq. (9) disturbs the state of neutral buoyancy. Since the initial temperature difference scales with $B^{2}$, this effect is more important for tubes with a large field strength (Moreno-Insertis 1983). On the other hand, for a tube radius larger than $1000 \mathrm{~km}$ the rise time

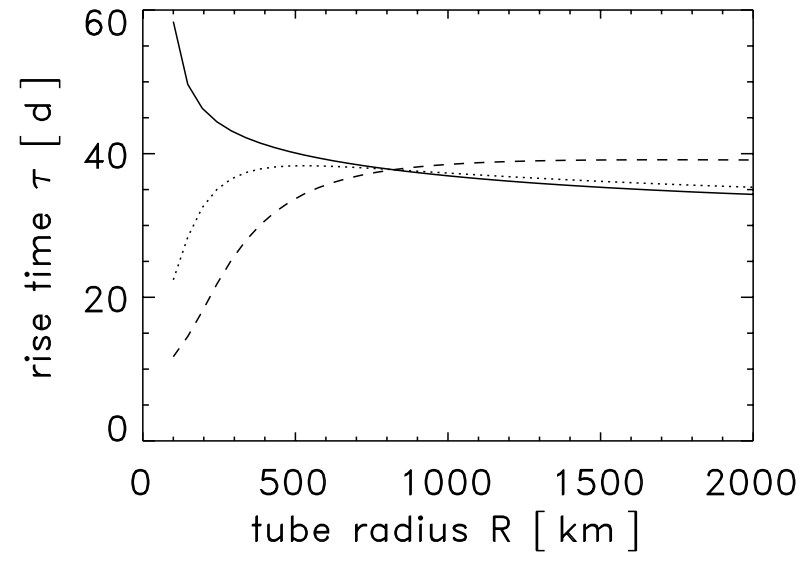

Fig. 1. Rise time of a flux ring over a radial distance of $10^{4} \mathrm{~km}$ within the overshoot region $\left(\delta=-3 \times 10^{-6}\right)$ as a function of the initial tube radius, $R$. The initial magnetic field strength is $B=1 \mathrm{~T}$ (solid line), $B=5 \mathrm{~T}$ (dotted), and $B=10 \mathrm{~T}$ (dashed). The simulation includes a drag force with $c_{w}=1$. The initial colatitude of the flux ring is $\theta_{0}=70^{\circ}$.

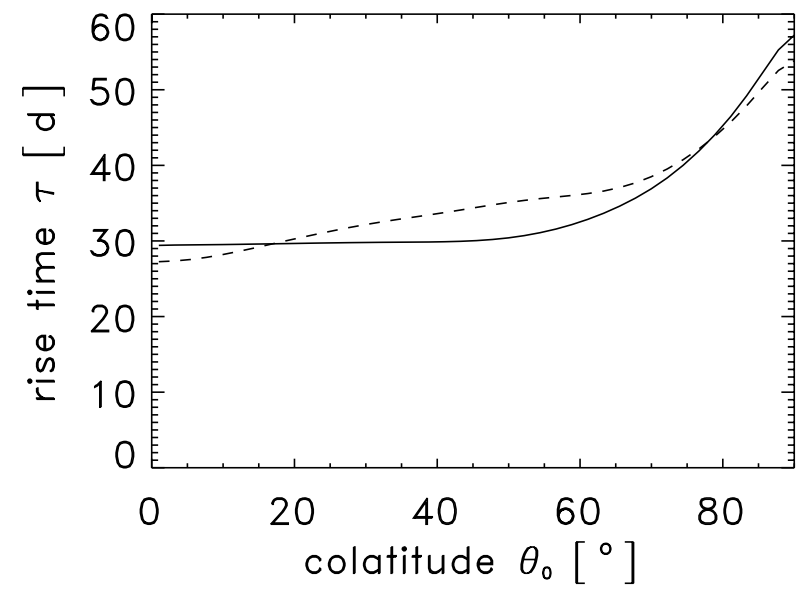

Fig. 2. Rise time of a flux ring over a radial distance of $10^{4} \mathrm{~km}$ within the overshoot region $\left(\delta=-3 \times 10^{-6}\right)$ as function of the initial colatitude. The tube radius is $1000 \mathrm{~km}$. The initial magnetic field strength is $B=1 \mathrm{~T}$ (solid line) and $B=10 \mathrm{~T}$ (dashed).

is nearly independent of the field strength since the heat exchange term scales with $R^{-2}$ (first term on the right-hand side of Eq. (9)) whereas the radiative heating term (second term on the right-hand side of Eq. (9)) shows no dependence on $R$.

Figure 2 shows the dependence of the rise time on the initial colatitude $\theta_{0}$ for a fixed initial tube radius of $1000 \mathrm{~km}$. The rise time roughly doubles for flux tubes starting near to the equatorial plane. This dependence can be understood in terms of angular momentum conservation, which tends to keep the distance of the flux ring to the axis of rotation constant. Whereas the buoyantly driven radial rise in high latitudes has nearly no effect on this distance, a rise near to the equator increases this distance, leading to a Coriolis force counteracting the radial rise. As a result the rise time increases towards low latitudes.

Figure 3 shows the rise time as function of the superadiabaticity, $\delta$. Since we have chosen a flux tube radius of $1000 \mathrm{~km}$, the influence of the field strength is small (Moreno-Insertis 1983). The linear dependence on $\delta$ predicted by Eq. (14) is 


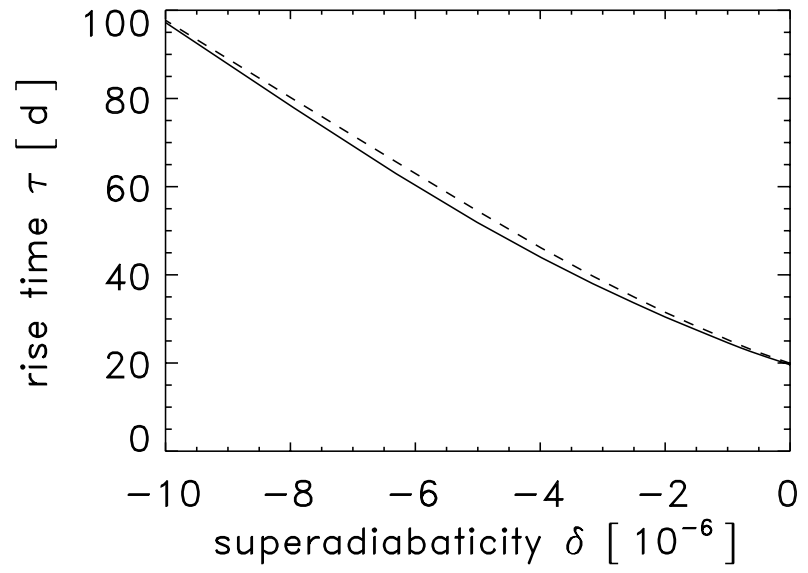

Fig. 3. Dependence of the rise time of a flux ring over a radial distance of $10^{4} \mathrm{~km}$ within the overshoot region on the superadiabaticity. The initial flux tube radius is $1000 \mathrm{~km}$, the initial colatitude $\theta_{0}=70^{\circ}$. The magnetic field strength is $B=1 \mathrm{~T}$ (solid) and $B=10 \mathrm{~T}$ (dashed).

confirmed, except for very small values of $|\delta|$, for which the $\beta$-dependence in Eq. (12) has to be taken into account.

\subsection{Influence of an external flow}

A meridional circulation with a poleward flow in the upper part of the solar convection zone has been found by helioseismology (e.g., Duvall \& Gizon 2000). If the return flow penetrates as deep as the overshoot layer, it may affect the evolution of magnetic flux tubes by dragging them equatorward. Angular momentum conservation then leads to an inward Coriolis force decelerating the radial rise. In order to simulate this effect we have included a $r$-independent equatorward flow of the form

$v_{\theta}^{m}(\theta)=v_{0} \sin (2 \theta)$,

which has a maximum speed at mid-latitudes. We will later discuss the influence of a flow profile with also radial dependence (see Eq. (31)). Figure 4 gives the resulting rise time for the same parameters as in Fig. 1 with a meridional flow with $v_{0}=20 \mathrm{~m} \mathrm{~s}^{-1}$ (Rekowski \& Rüdiger 1998). For flux tubes with radius larger than $\simeq 1000 \mathrm{~km}$, the rise time increases by about $30 \%$ compared to the case without meridional flow, largely independent of the field strength. For smaller radii, the rise time depends on the field strength: whereas flux tubes with $5 \mathrm{~T}$ and $10 \mathrm{~T}$ show rise times similar to those in Fig. 1, the rise time sharply increases for $B=1 \mathrm{~T}$. This result due to the fact that flux tubes with weak field and small radius find an equilibrium state that is characterized by a balance between the buoyancy force and the sum of the Coriolis and drag forces (van Ballegooijen 1982). We discuss the properties of such equilibria in the following section.

\subsection{Equilibria with meridional flow}

For an equilibrium with meridional flow and radiative heating the conditions for mechanical $(\ddot{r}=\dot{r}=\ddot{\theta}=\dot{\theta}=0$ ) and thermal equilibrium $(\dot{S}=0)$ have to be fulfilled simultaneously.

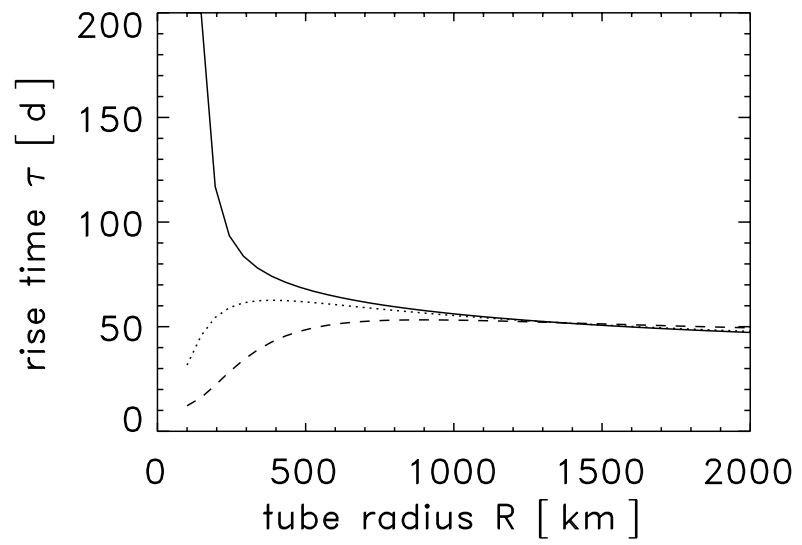

Fig. 4. Same as in Fig. 1, but including an external equatorward meridional flow with a velocity of $20 \mathrm{~m} \mathrm{~s}^{-1}$. For flux tubes with small radius and weak field, the meridional flow leads to a significantly larger rise time.

\section{Thermal equilibrium:}

The thermal equilibrium requires a vanishing net heating rate of the flux tube. Setting the right-hand side of Eq. (9) equal to zero, we obtain for the temperature difference:

$\frac{\Delta T}{T} \equiv \frac{T_{\mathrm{e}}-T}{T}=\frac{-\operatorname{div} \boldsymbol{F}_{\mathrm{e}}}{4 \kappa T} R^{2}$.

With $\operatorname{div} \boldsymbol{F}_{\mathrm{e}} \approx-3 \mathrm{~W} \mathrm{~m}^{-3}$, thermal equilibrium requires a larger temperature inside the flux tube:

$\Delta T \simeq 80\left(\frac{R}{1000 \mathrm{~km}}\right)^{2} \mathrm{~K}$

\section{Radial force balance:}

Assuming $\beta \gg 1$ we can express the growth of the field strength due to stretching of the expanding flux ring in the form

$\frac{B}{\varrho}=\frac{B_{0}}{\varrho_{0}} \frac{r \sin \theta}{r_{0} \sin \theta_{0}} \equiv \frac{B_{0}}{\varrho_{0}} x$,

while the change of the internal azimuthal flow due to angular momentum conservation is given by

$\dot{\phi}=\Omega\left(\frac{1}{x^{2}}-1\right)$

Inserting Eqs. (19) and (20) together with the initial condition Eq. (11) into Eqs. (1) and (4) yields for the equilibrium position $(\dot{\theta}=\dot{r}=\ddot{r}=0)$ :

$$
\begin{aligned}
\frac{\Delta \varrho}{\varrho}= & {\left[\Omega^{2} r \sin ^{2} \theta\left(\frac{1}{x^{4}}-1\right)-\frac{B^{2}}{\mu_{0} \varrho r}\left(1-\frac{\varrho}{\varrho}\right)\right] } \\
& \cdot \frac{1}{g-\Omega_{\mathrm{e}}^{2} r \sin ^{2} \theta},
\end{aligned}
$$

where we assumed a purely latitudinal external flow $\left(v_{\perp r}=0\right)$.

For solar rotation we have $\Omega_{\mathrm{e}}^{2} r \sin ^{2} \theta \ll g$. Excluding the vicinity of the poles, the first term in angular brackets dominates over the second term. For $\theta=70^{\circ}, B=5 \mathrm{~T}$, 
and $\varrho / \varrho_{0}=0.9$ (which corresponds to a radial displacement of $\Delta r=10^{4} \mathrm{~km}$ ) we have

$\Omega^{2} r \sin ^{2} \theta \sim 3 \times 10^{-3}$

$\frac{B^{2}}{\mu_{0} \varrho r}\left(1-\frac{\varrho_{0}}{\varrho}\right) \sim 2 \times 10^{-5}$.

As long as the flux tube remains in the overshoot region, we have $x \lesssim 1.02$, which corresponds to a radial displacement of $\Delta r=10^{4} \mathrm{~km}$ at $r=5 \times 10^{8} \mathrm{~m}$. This means that the first term in angular brackets in Eq. (21) is a factor of 10 larger than the second term. From the linearized ideal gas law together with the lateral balance of total pressure we obtain

$\frac{\Delta \varrho}{\varrho}=-\frac{1}{\beta}-\frac{\Delta T}{T}$

Inserting this into Eq. (21), we find the equilibrium field strength, $B_{\text {eq }}$ :

$B_{\text {eq }}=\sqrt{2 \mu_{0} p\left[\frac{\Omega^{2} r \sin ^{2} \theta}{g}\left(1-\frac{1}{x^{4}}\right)-\frac{\Delta T}{T}\right]}$,

where $p$ denotes the gas pressure within the flux tube.

\section{Latitudinal force balance:}

The external meridional flow enters the equations through the drag term in the $\theta$-component of the momentum equation, Eq. (2). Setting $\ddot{\theta}=0$ and using Eqs. (19) and (20) we obtain as equilibrium condition:

$$
\begin{aligned}
0= & \frac{B^{2}}{\mu_{0} \varrho r}\left(1-\frac{\varrho_{0}}{\varrho}\right) \cot \theta-\frac{\Delta \varrho}{\varrho} \Omega_{\mathrm{e}}^{2} r \sin \theta \cos \theta \\
& +\Omega^{2} r \sin \theta \cos \theta\left(\frac{1}{x^{4}}-1\right)+\frac{c_{w}}{\pi R} \frac{\varrho_{\mathrm{e}}}{\varrho}\left(v_{\theta}^{m}\right)^{2} .
\end{aligned}
$$

The first term on the right-hand side can be neglected as shown above in the case of the radial component. We see from Eq. (21) that the second term is by a factor $\Omega_{\mathrm{e}}^{2} r / g \approx 10^{-5}$ smaller than the third term and thus is negligible as well. With Eq. (16) the condition for the latitudinal force equilibrium then reads

$1-\frac{1}{x^{4}}=\frac{2}{\pi} \frac{c_{w} v_{0}^{2}}{\Omega^{2} r R} \sin (2 \theta)$.

We see from Eq. (11) that $\Omega$ depends on the initial position of the flux tube and on its initial field strength, $B_{0}$. In general it is not possible to obtain an analytical solution for $x$.

Since the difference between $\Omega$ and $\Omega_{\mathrm{e}}$ is small in the relevant range of field strengths $\left(<10 \%\right.$ for a $5 \mathrm{~T}$ field and $\left.\theta>30^{\circ}\right)$, an approximate solution of Eq. (27) can be found by setting $\Omega=\Omega_{\mathrm{e}}$. For equatorward flow in the overshoot region we always have $x>1$, so that there exists a minimal equilibrium tube radius, $R_{\min }$. In the limiting case $x \rightarrow \infty$ (so that $B_{0} \rightarrow 0$ and thus $\Omega \rightarrow \Omega_{\mathrm{e}}$ ) we have

$R_{\min }=\frac{2}{\pi} \frac{c_{w} v_{0}^{2}}{\Omega_{\mathrm{e}}^{2} r} \sin (2 \theta)$.

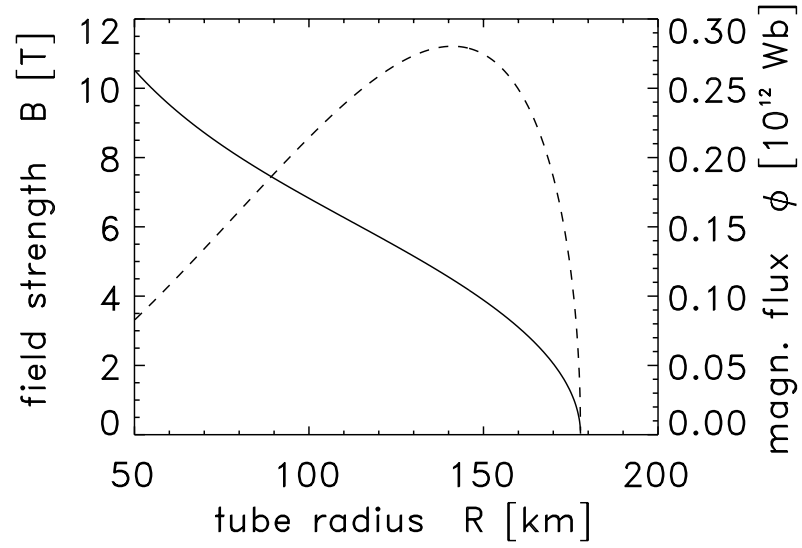

Fig. 5. Field strength (solid line, left scale) and magnetic flux (dashed line, right scale) for an equilibrium flux ring located at $\theta=70^{\circ}$ colatitude. The meridional flow has an amplitude of $v_{0}=20 \mathrm{~m} \mathrm{~s}^{-1}$. The values of $R_{\min }$ and $R_{\max }$ are $50 \mathrm{~km}$ and $178 \mathrm{~km}$, respectively.

For flux tubes with $R<R_{\min }$, the Coriolis force cannot balance the drag force and stop the equatorward drift due to the latitudinal flow. Combining Eqs. (17), (25), and (27) yields

$B_{\mathrm{eq}}=\sqrt{2 \mu_{0} p\left(\frac{2}{\pi} \frac{c_{w} v_{0}^{2} \sin ^{2} \theta \sin (2 \theta)}{g R}-\frac{\left|\operatorname{div} \boldsymbol{F}_{\mathrm{e}}\right|}{4 \kappa T} R^{2}\right)}$.

Since this equation does not contain $\Omega$, there is no implicit dependence on the initial state. Equation (29) implies a maximum tube radius compatible with the equilibrium:

$R<R_{\max }=\left(\frac{8}{\pi} \frac{T \kappa c_{w} v_{0}^{2} \sin ^{2} \theta \sin (2 \theta)}{g\left|\operatorname{div} \boldsymbol{F}_{\mathrm{e}}\right|}\right)^{1 / 3}$.

For flux tubes with larger radius, the buoyancy force caused by the temperature difference given by Eq. (17) cannot be balanced by the Coriolis and drag forces. Thus equilibria only exist in the range $R_{\min }<r<R_{\max }$. In this interval, the equilibrium field strength is a function of tube radius and latitudinal flow speed. Figure 5 shows $B_{\text {eq }}$ and the corresponding magnetic flux as a function of tube radius for $v_{0}=20 \mathrm{~m} \mathrm{~s}^{-1}$.

Figure 6 shows the dependence of $R_{\min }$ and $R_{\max }$ on the meridional flow speed, $v_{0}$. Equilibria only exist for $R_{\min } \leq$ $R_{\text {max }}$, which implies $v_{0} \leq 50 \mathrm{~ms}^{-1}$. Returning to the results shown in Fig. 4, we see that the strong increase of the rise time for the tube with $B=1 \mathrm{~T}$ can be understood as the result of the approach towards the interval of possible equilibrium configurations $\left(R<178 \mathrm{~km}\right.$ for $\left.v_{0}=20 \mathrm{~m} \mathrm{~s}^{-1}\right)$.

After discussing the equilibria we now consider whether such a state can actually be reached dynamically. Equation (29) shows that, for a given flow speed $v_{0}$ and colatitude $\theta$, the equilibrium requires a specific relation between $B$ and $R$. Since it is not obvious that a given flux tube will reach an equilibrium state, we have carried out numerical simulations focusing on the evolution towards a state of equilibrium. For a meridional flow driven by the interaction of convection and rotation in the convection zone, we expect that flow speed decreases with depth in the overshoot region (e.g., Rekowski \& Rüdiger 1998). 


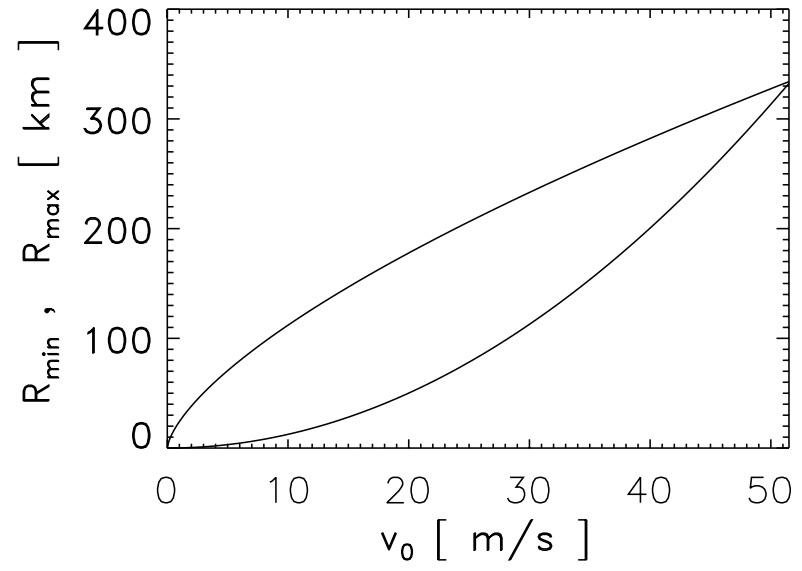

Fig. 6. The interval $\left[R_{\min }, R_{\max }\right]$ for equilibrium flux tubes as a function of the meridional flow speed. Equilibria exist only for $v_{0}<50 \mathrm{~m} \mathrm{~s}^{-1}$. For larger flow speeds, the conditions for radial and lateral force balance cannot be fulfilled simultaneously.

In the attempt to mimic this effect, we have used the velocity profile

$v_{\theta}^{m}(r, \theta)=v_{0} \sin (2 \theta)\left[1-\exp \left(-\frac{\left(r-r_{0}\right)^{2}}{d^{2}}\right)\right]$,

with $d=4 \times 10^{6} \mathrm{~m}$. The flow speed vanishes at the bottom of the overshoot region, $r=r_{0}$. The results of the simulation with this velocity profile depend on whether the initial field strength and initial radius of the tube permit an equilibrium configuration according to Eqs. (28)-(30):

1) If no equilibrium can exist for a meridional flow speed with $v \leq v_{0}$, the flux tube leaves the overshoot region.

2) If an equilibrium with $v \leq v_{0}$ is possible, the flux tube drifts equatorward and in radial direction until it encounters the value of $v_{\theta}^{m}$ required for equilibrium.

An example for the dynamic evolution of a flux tube towards an equilibrium is shown in Fig. 7. Initially, the thermal buoyancy due to radiative heating leads to a radial upward drift of about $2.5 \mathrm{Mm}$ while the latitudinal flow displaces the flux tube towards the equator by about $12^{\circ}$. The toroidal flow speed inside the flux tube decreases due to angular momentum conservation. The resulting Coriolis force and the drag force more than compensate the thermal buoyancy, so that the flux tube drifts downward again until it reaches the depth in which the meridional flow speed permits force equilibrium. In its equilibrium position, the flux tube reaches a field strength of $B=4 \mathrm{~T}$ and a radius of $R=150 \mathrm{~km}$. The latitudinal flow speed of $v_{0}=20 \mathrm{~m} \mathrm{~s}^{-1}$ at this position corresponds to the case shown in Fig. 5.

\section{Radiative heating of a magnetic layer}

So far we have discussed the consequences of a non-vanishing $\operatorname{div} \boldsymbol{F}_{\mathrm{e}}$ in the case of isolated flux tubes. For a homogeneous magnetic field layer, the resulting heat exchange affects the stratification of the whole overshoot region. Depending on its width and radial location, the magnetic layer may straddle regions with different sign of $\operatorname{div} \boldsymbol{F}_{\mathrm{e}}$. For instance, we have
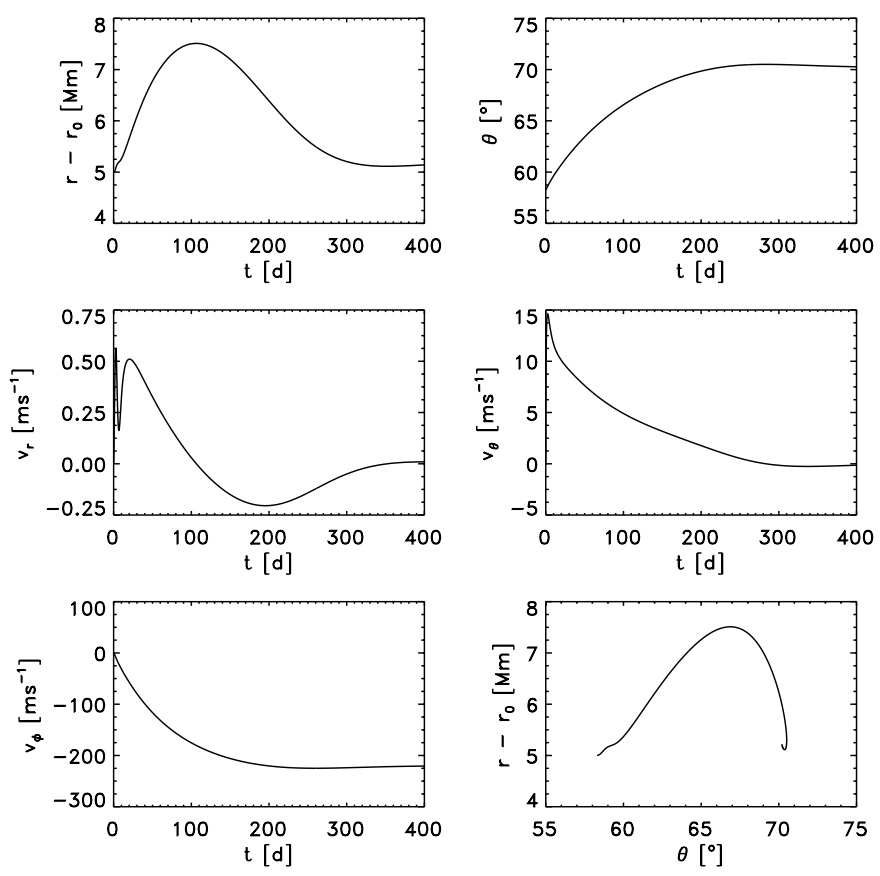

Fig. 7. Evolution of a flux ring towards an equilibrium state with $B=4 \mathrm{~T}$ and $R=150 \mathrm{~km}$. The meridional flow has a maximum value of $v_{0}=25 \mathrm{~m} \mathrm{~s}^{-1}$ and decreases with depth according to Eq. (31). Shown is the development of radial distance from bottom of overshoot region (upper left panel), colatitude (upper right), and the three velocity components (middle left, middle right, lower left panel). The lower right panel shows the path of the flux ring in the $r-\theta$ plane.

$\operatorname{div} \boldsymbol{F}_{\mathrm{e}}>0$ (and thus a cooling effect) in the narrow boundary layer at the bottom of the overshoot region where the downward directed convective energy flux decreases to zero.

We consider a simple one-dimensional model for the thermal effects arising from the suppression of convection within the magnetic layer. We assume a linear diffusion model for the convective smoothing of temperature perturbations (Spruit 1977a; Moreno-Insertis et al. 2002) in order to describe the reaction of the temperature profile on a magnetic quenching of the convective flux. This allows us to calculate the change of superadiabaticity and thus to determine how the conditions for flux storage are changed by thermal effects. The model does not describe the backreaktion of the superadiabaticity on the convection itself. However, the results do not depend sensitively on the details of the quenching since the solution is mainly determined by the assumed magnitude of the reduction of the convective heat flux.

\subsection{Model equations}

The undisturbed solar energy flux in the solar overshoot region consists of a convective and radiative part,

$\boldsymbol{F}=\underbrace{\alpha \boldsymbol{F}_{\odot}}_{\boldsymbol{F}_{\mathrm{r}}}+\underbrace{(1-\alpha) \boldsymbol{F}_{\odot}}_{\boldsymbol{F}_{\mathrm{c}}}$,

with $\alpha>1$ because the (non-locally driven) convective energy flux is directed downward in the overshoot region. We account for the suppression of the convective energy flux by a strong 
magnetic field by introducing a flux quenching factor, $q_{\mathrm{f}}$, defined by

$\boldsymbol{F}_{\mathrm{c}}=q_{\mathrm{f}} \boldsymbol{F}_{\mathrm{c}}^{0}$

with the unperturbed convective energy flux $\boldsymbol{F}_{\mathrm{c}}^{0}$. The reduced convective energy flux leads to a temperature perturbation, $T_{1}$. Following Moreno-Insertis et al. (2002), we assume that the effect of the convective motions on $T_{1}$ can be described as a local diffusion process, analogous to the effect of radiative transport. Owing to the presence of the magnetic field, the corresponding convective heat conductivity is also subject to a quenching factor, $q_{\kappa}$, defined by

$\kappa_{\mathrm{c}}=q_{\kappa} \kappa_{\mathrm{c}}^{0}$.

The magnetically modified energy flux is thus written as:

$$
\begin{aligned}
\boldsymbol{F}= & \boldsymbol{F}_{\odot}-\left(\kappa_{\mathrm{r}}+q_{\kappa} \kappa_{\mathrm{c}}^{0}\right) \operatorname{grad} T_{1} \\
& +\boldsymbol{F}_{\odot}\left[\alpha \frac{\kappa_{\mathrm{r}}^{1}}{\kappa_{\mathrm{r}}^{0}}-(1-\alpha)\left(1-q_{\mathrm{f}}\right)\right]
\end{aligned}
$$

where $\kappa_{\mathrm{r}}^{0}$ and $\kappa_{\mathrm{c}}^{0}$ denote the unperturbed radiative and convective heat conductivities. $\kappa_{\mathrm{r}}^{1}$ is the perturbation of the radiative conductivity and $\kappa_{\mathrm{r}}=\kappa_{\mathrm{r}}^{0}+\kappa_{\mathrm{r}}^{1}$. In general, $q_{\mathrm{f}}$ and $q_{\kappa}$ are expected to be different because of the non-locality of the background convective energy flux. The relation between both would result from a detailed convection theory including the influence of the magnetic field, which is not available. However, in the limit of strong quenching of the convective energy flux $\left(0 \leq q_{\mathrm{f}} \ll 1\right)$, Eq. (35) becomes largely independent of $q_{\mathrm{f}}$, so that only $q_{\kappa}$ affects the solution. We restrict ourselves to this case. A further simplification can be achieved by observing that the relative disturbance of the radiative conductivity, $\kappa_{\mathrm{r}}^{1} / \kappa_{\mathrm{r}}^{0}$, is of the order of the relative temperature disturbance, $T_{1} / T_{0} \ll 1$. For $q_{\mathrm{f}} \ll 1$ and $\alpha \neq 1$, the first term in the angular brackets in Eq. (35) is thus much smaller than the second term and may be neglected. With these simplifications, the equation for the time evolution of the temperature disturbance reads:

$$
\begin{aligned}
\varrho c_{\mathrm{p}} \frac{\partial T_{1}}{\partial t}= & \operatorname{div}\left[\left(\kappa_{\mathrm{r}}+q_{\kappa} \kappa_{\mathrm{c}}^{0}\right) \operatorname{grad} T_{1}\right] \\
& +\boldsymbol{F}_{\odot} \operatorname{grad}\left[(1-\alpha)\left(1-q_{\mathrm{f}}\right)\right] .
\end{aligned}
$$

The last expression on the right-hand side is analogous to the term $-\operatorname{div} \boldsymbol{F}_{\mathrm{e}}$ in Eq. (9) for an isolated flux tube. In the case of totally suppressed convection $\left(q_{\mathrm{f}}=0\right)$, this term is given by

$H_{\mathrm{rad}}=-F_{\odot} \frac{\mathrm{d} \alpha}{\mathrm{d} r}$,

where $F_{\odot}=\left|\boldsymbol{F}_{\odot}\right|$. The gradient of $\alpha$ is nearly constant over most of the overshoot region, so that we have a nearly constant heating rate in regions with suppressed convection. Near to the boundaries of the magnetic layer, $q_{\mathrm{f}}$ varies strongly, so that we have an additional contribution:

$H_{\mathrm{rad}}^{\prime}=-F_{\odot}(1-\alpha) \frac{\mathrm{d} q_{\mathrm{f}}}{\mathrm{d} r}$.

This term leads to localized heating (or cooling) near the edges of the magnetic layer; it describes the formation of thermal shadows above and below the layer. The sign of this term depends on $1-\alpha$, being opposite in the overshoot region $(\alpha>1)$ and the convection zone proper $(\alpha<1)$.

We assume a thin magnetic layer with a much larger horizontal than radial extension, so that the horizontal deflection of the convective energy flux can be neglected. In this case, a onedimensional description only considering the radial component of the heat flux is sufficient, which simplifies Eq. (36):

$$
\begin{aligned}
\varrho c_{\mathrm{p}} \frac{\partial T_{1}}{\partial t}= & \frac{1}{r^{2}} \frac{\mathrm{d}}{\mathrm{d} r}\left[r^{2}\left(\kappa_{\mathrm{r}}+q_{\kappa} \kappa_{\mathrm{c}}^{0}\right) \frac{\mathrm{d} T_{1}}{\mathrm{~d} r}\right] \\
& +F_{\odot} \frac{\mathrm{d}}{\mathrm{d} r}\left[(1-\alpha)\left(1-q_{\mathrm{f}}\right)\right] .
\end{aligned}
$$

In order to determine the change of the superadiabaticity arising from the temperature perturbation, we have to determine the pressure perturbation, $p_{1}$. Since the time scale for hydrostatic equilibrium in the overshoot region (of a few minutes) is much shorter than the time scale for the temperature change described by Eq. (36) (of the order of months) we can assume quasi-static changes. The total pressure disturbance $\pi_{1}=p_{1}+B^{2} / 2 \mu_{0}$ then results from the equation of (magneto-)hydrostatic equilibrium,

$\frac{\mathrm{d} \pi_{1}}{\mathrm{~d} r}=-\varrho_{1} g$,

together with the linearized ideal gas law,

$\varrho_{1}=\varrho_{0}\left(\frac{p_{1}}{p_{0}}-\frac{T_{1}}{T_{0}}\right)$.

This leads to

$\frac{\mathrm{d} \pi_{1}}{\mathrm{~d} r}=-\frac{\pi_{1}}{H_{p 0}}+\varrho_{0} g\left(\frac{T_{1}}{T_{0}}+\frac{B^{2}}{2 \mu_{0} p_{0}}\right)$,

where $H_{p 0}=p_{0} /\left(\varrho_{0} g\right)$ is the undisturbed pressure scale height. As boundary condition we take $p_{1}=0$ at a radial position deep enough within the radiation zone, so that the stratification remains unaffected by the thermal changes caused by the magnetic layer. For solving Eq. (42) we assume that the magnetic field remains unchanged and only the gas pressure adjusts to the change of the temperature profile. Once $\pi_{1}(r)$ is determined, the perturbation of the superadiabaticity, $\delta_{1}$, is given by

$\delta_{1}=H_{p 0}\left(\frac{1}{\varrho_{0}} \frac{\mathrm{d} \varrho_{1}}{\mathrm{~d} r}-\frac{1}{\gamma p_{0}} \frac{\mathrm{d} p_{1}}{\mathrm{~d} r}-\frac{\varrho_{1}}{\varrho_{0}^{2}} \frac{\mathrm{d} \varrho_{0}}{\mathrm{~d} r}+\frac{p_{1}}{\gamma p_{0}^{2}} \frac{\mathrm{d} p_{0}}{\mathrm{~d} r}\right)$.

\subsection{Numerical results}

We solve Eq. (39) by using a semi-implicit finite difference scheme for the diffusion operator and a typical number of 1000 equidistant grid points for a radial extension of $5 \times 10^{7} \mathrm{~m}$. For a quantitative evaluation of the model, we consider a case in which $\alpha$ and $\kappa_{\mathrm{c}}^{0}$ are prescribed by analytical functions approximating the results from the non-local mixing-length model of Skaley \& Stix (1991):

$\alpha= \begin{cases}1 & \text { for } r<r_{0} \\ 1+\frac{\left(r-r_{0}\right) / d_{1}}{1+\left(r-r_{0}\right) / d_{1}} \cdot & \text { for } r>r_{0} \\ {\left[(1+\tilde{\alpha}) \exp \left(-\frac{r-r_{0}}{d_{2}}\right)-1\right]}\end{cases}$

$\kappa_{\mathrm{c}}^{0}= \begin{cases}0 & \text { for } r<r_{0} \\ c \sqrt{\left(r / r_{0}\right)^{2}-1} & \text { for } r>r_{0} .\end{cases}$ 

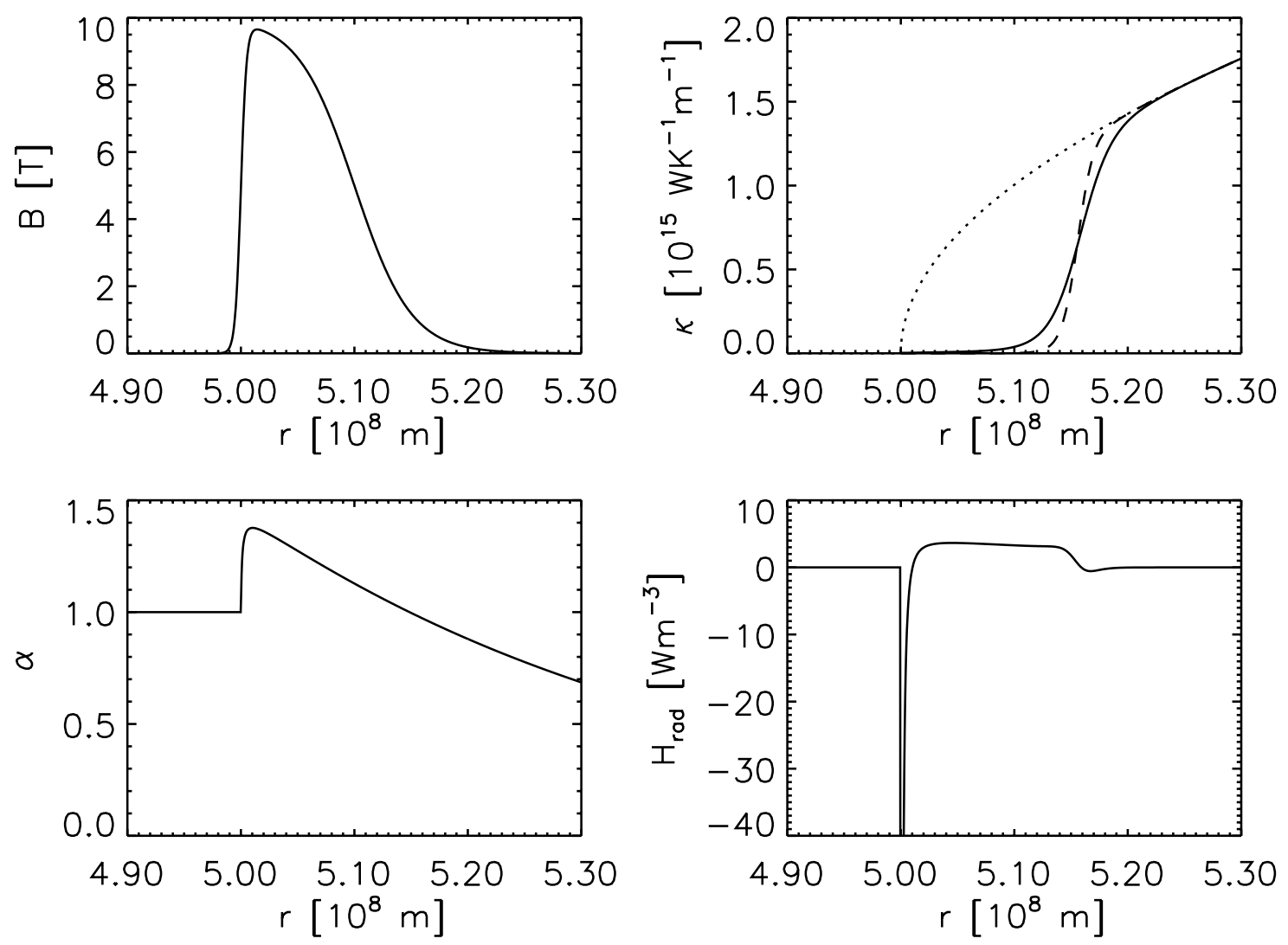

Fig. 8. Radial dependence of quantities used in the numerical calculations for magnetic layers in the overshoot region. Top left: magnetic field strength. Bottom left: ratio of radiative to total energy flux $\left(\alpha=F_{\mathrm{r}} / F_{\odot}\right)$. Top right: convective heat conductivity, $\kappa_{\mathrm{c}}$, from Eq. (47) for $\epsilon=2$ (solid curve) and $\epsilon=4$ (dashed curve). The dotted curve shows the undisturbed heat conductivity, $\kappa_{\mathrm{c}}^{0}$. Bottom right: radiative heating rate. The cooling near the base of the overshoot region caused by the strong increase of $\alpha$ reaches a peak value of about $-250 \mathrm{~W} \mathrm{~m}^{-3}$, whereas the heating within the overshoot region has a nearly constant value of $3 \mathrm{~W} \mathrm{~m}^{-3}$.

The parameter $\tilde{\alpha}$ characterizes the vigor of the overshoot, $d_{1}$ is the width of the transition towards the radiation zone at the base of the overshoot region, and $d_{2}$ represents the length scale of the decrease of $\alpha$ in the overshoot region and convection zone. The parameter $c$ determines the magnitude of the convective thermal conductivity. Figure 8 shows the profiles of $\alpha$ and $\kappa_{\mathrm{c}}^{0}$ for $\tilde{\alpha}=0.45, d_{1}=100 \mathrm{~km}, d_{2}=4 \times 10^{4} \mathrm{~km}$, $c=5 \times 10^{15} \mathrm{~W} \mathrm{~K}^{-1} \mathrm{~m}^{-1}$ and $r_{0}=5 \times 10^{8} \mathrm{~m}$, together with the field strength in the magnetic layer as given by the function

$$
\begin{gathered}
B(r)=\frac{1}{4} B_{0}\left[1+\tanh \left(\frac{r-r_{B 1}}{d_{B 1}}\right)\right] . \\
{\left[1-\tanh \left(\frac{r-r_{B 2}}{d_{B 2}}\right)\right] .}
\end{gathered}
$$

The parameter values used in Fig. 8 are $B_{0}=10 \mathrm{~T}$ for the maximum field strength, $r_{B 1}=5 \times 10^{8} \mathrm{~m}, d_{B 1}=5 \times 10^{5} \mathrm{~m}$, $r_{B 2}=5.1 \times 10^{8} \mathrm{~m}$, and $d_{B 2}=5 \times 10^{6} \mathrm{~m}$. In analogy to quenching approaches in turbulent dynamo theory (e.g., Tobias 1996), we assume for the magnetic quenching of the convective heat conductivity the relationship

$\kappa_{\mathrm{c}}=q_{\kappa} \kappa_{\mathrm{c} 0}=\left[1+\left(\frac{B}{B_{\mathrm{eq}}}\right)^{\epsilon}\right]^{-1} \kappa_{\mathrm{c} 0}$,

where $B_{\text {eq }}$ denotes the equipartition field strength with respect to the kinetic energy density of the convective motions and the parameter $\epsilon$ parameterizes the strength of the quenching. We use values of $B_{0}=10 \mathrm{~T}$ and $B_{\mathrm{eq}}=1 \mathrm{~T}$ corresponding to the expected conditions in the solar overshoot layer. Calculations have been carried out for $\epsilon=2$ (suppression of $\kappa_{\mathrm{c}}$ by a factor of $10^{2}$ ) and $\epsilon=4$ (suppression by a factor of $10^{4}$ ). With $\kappa_{\mathrm{c}}^{0} \simeq 10^{15} \mathrm{~W} \mathrm{~K}^{-1} \mathrm{~m}^{-1}$ and $\kappa_{\mathrm{r}} \simeq 10^{10} \mathrm{~W} \mathrm{~K}^{-1} \mathrm{~m}^{-1}$ (Spruit 1977a) this means that the convective heat conductivity in the magnetic layer has values of $\kappa_{\mathrm{c}} \simeq 10^{3} \kappa_{\mathrm{r}}$ and $\kappa_{\mathrm{c}} \simeq 10 \kappa_{\mathrm{r}}$, respectively. The corresponding thermal diffusion time scale

$\tau_{\text {diff }}=\frac{d^{2} \varrho c_{\mathrm{p}}}{\kappa_{\mathrm{c}}}$

has values of 2 and 200 years, respectively, assuming a thickness of the magnetic layer of $d=10^{4} \mathrm{~km}$.

The radiative heating rate given by the second term on the right-hand side of Eq. (39) yields a nearly depth-independent heating of approximately $3 \mathrm{Wm}^{-3}$ within the overshoot region and very strong cooling in the narrow boundary region towards the radiation zone (see Fig. 8). The amplitude of the cooling peak depends sensitively on the width of the boundary region, but has no significant influence on the overall result since the volume-integrated cooling rate is fixed by the jump of $\alpha$. The result is determined mainly by the strength of the magnetic quenching of the convective heat conductivity, which enters Eq. (39) mainly through the first (diffusive) 

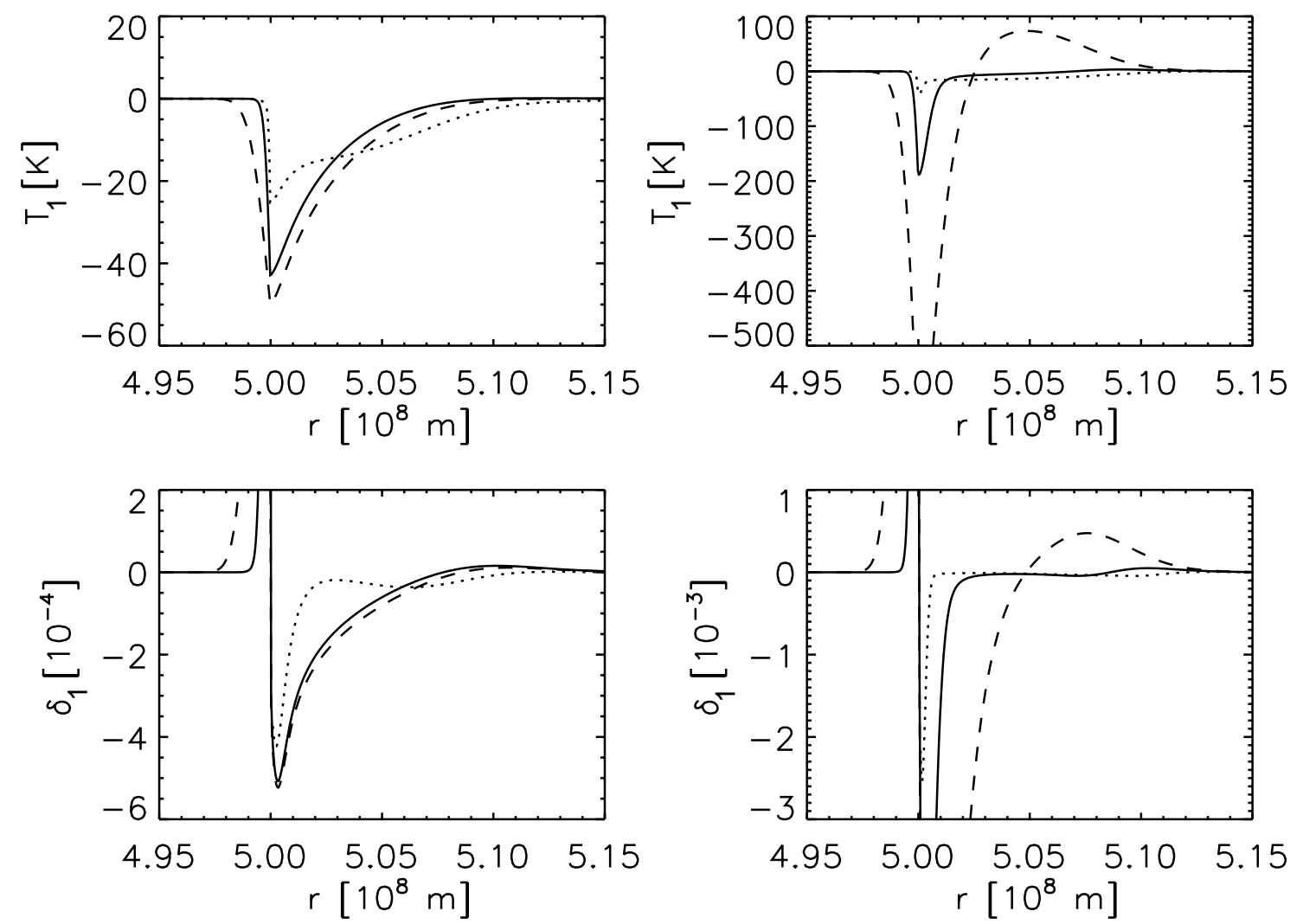

Fig. 9. Time evolution of the perturbations of temperature and superadiabaticity for a quenching of the convective heat conductivity by a factor of $10^{2}\left(\epsilon=2\right.$, left) and $10^{4}(\epsilon=4$, right), respectively. The curves correspond to $t=1$ month (dotted), $t=6$ months (solid), and $t=5$ years (dashed) elapsed time. Note that the disturbances in the latter case are by an order of magnitude larger. In the case $\epsilon=2$, the temperature disturbance in the region $r>5 \times 10^{8} \mathrm{~m}$ approaches the asymptotic profile already after a few months. The significant decrease of the superadiabaticity above $r=5 \times 10^{8} \mathrm{~m}$ shows that the change of the temperature profile stabilizes the stratification. In the case $\epsilon=4$, the quenched convective heat conductivity is insufficient to couple the regions with radiative heating and cooling, respectively, so that strong perturbations arise. In this case, the upper part of the magnetic layer is heated and destabilized. Note that we only show the perturbation of the superadiabaticity, $\delta_{1}$. The background stratification has values of $\delta_{0}$ in the range $-10^{-6} \ldots-10^{-5}$ in the overshoot region and about -0.2 in the radiation zone.

term on the right-hand side, whereas the radiative heating term is nearly independent of the magnetic field as long as $\left(q_{\mathrm{f}} \ll 1\right)$.

In order to simulate the time evolution we solve Eq. (39) using a finite-difference scheme with semi-implicit treatment of the diffusion term. We calculate the perturbation of the superadiabaticity in each time step by solving Eqs. (42) and (43). The resulting evolution of the temperature and superadiabaticity perturbations are shown in Fig. 9 for the cases $\epsilon=2$ and $\epsilon=4$, respectively. In the first case, the convective heat conductivity is still large enough for efficient heat exchange within a few months between the regions with radiative heating and cooling. The resulting temperature perturbation is negative in the overshoot region, reaching a peak value of about $-50 \mathrm{~K}$. This stabilizes the stratification by lowering the superadiabaticity to values of a few times $-10^{-4}$. The part of the solution within the radiation zone corresponds to a traveling cooling front, which does not reach a stationary state within the evolution time considered here. We show in the section below that the influence of this cooling front on the temperature profile in the overshoot region is negligible.

The situation is different in the case $\epsilon=4$, corresponding to a suppression of the convective heat conductivity by a factor of $10^{4}$. Such a strongly quenched heat conductivity cannot prevent the development of strong temperature perturbations, which initially follow the profile of the radiative heating: the upper part of the magnetic layer is heated whereas the lowermost part is strongly cooled. As a consequence, the upper half of the overshoot region is destabilized $(\delta>0)$, so that probably the magnetic layer cannot be maintained there. The asymptotic solution shows a profile similar to the case $\epsilon=2$, but it requires roughly a (diffusion) time of about 200 years to reach this state. Thus this solution is irrelevant for magnetic fields varying in the course of the 11-year solar cycle.

\subsection{Analytical model}

In the special case of a piecewise constant radial profile of the total heat conductivity and a constant radiative heating rate within the overshoot region, we obtain an analytical expression for the asymptotic solution of the diffusion problem in the overshoot region and also for the traveling cooling front in the radiation zone. Since the thickness of the overshoot region is small compared to the solar radius, geometrical factors following from the spherical geometry are neglected. Furthermore, 
we assume that the transition between overshoot region and radiation zone is sharp. The total heat conductivity $\kappa=\kappa_{\mathrm{r}}+q_{\kappa} \kappa_{\mathrm{c}}^{0}$ is a piecewise constant function of radius:

$\kappa=\left\{\begin{aligned} r<r_{0}: & \kappa=\kappa_{\mathrm{r}} \\ r_{0}<r<r_{1}: & \kappa=\tilde{\kappa} \\ r>r_{1}: & \kappa=\kappa_{\mathrm{c}}^{0}\end{aligned}\right.$

with $\kappa_{\mathrm{r}}<\tilde{\kappa}<\kappa_{\mathrm{c}}^{0}$. The interval $\left[r_{0}, r_{1}\right]$ represents the magnetic layer. In the radiation zone, $r<r_{0}$, the solution is a traveling cooling front. Ignoring the geometrical factors $\left(r^{2}\right)$ in Eq. (39) admits a similarity solution given by

$T_{1}(r, t)=T_{1}^{0}[1-2 \Phi(\xi)]$,

where

$\xi=\left(r_{0}-r\right) \sqrt{\frac{\varrho c_{\mathrm{p}}}{2 \kappa_{\mathrm{r}} t}}$

and $\Phi(\xi)$ denotes the error function. In the case $\tilde{\kappa} \gg \kappa_{\mathrm{r}}$, the solution between $r_{0}$ and $r_{1}$ quickly approaches an asymptotic state with a parabolic temperature profile determined by the constant heating rate, $H_{\text {rad }}$, in this region:

$T_{1}=T_{1}^{0}+a\left(r-r_{0}\right)-\frac{1}{2} \frac{H_{\mathrm{rad}}}{\tilde{\kappa}}\left(r-r_{0}\right)^{2}$.

The constants $a$ and $T_{0}^{1}$ are determined by requiring the continuity of temperature and heat flux at $r_{0}$ and $r_{1}$. The continuity of the heat flux at $r=r_{0}$ leads to (see Eq. (35))

$\tilde{\kappa}\left[\frac{\mathrm{d} T_{1}}{\mathrm{~d} r}\right]_{r_{0+}}-\kappa_{r}\left[\frac{\mathrm{d} T_{1}}{\mathrm{~d} r}\right]_{r_{0-}}=F_{\odot} \alpha_{0}$,

where $\alpha_{0}$ denotes the magnitude of the jump of $\alpha$ at the base of the overshoot region. Using Eq. (50), the radiative heat flux perturbation at the top of the radiation zone $\left(r<r_{0}\right)$ is given by

$\kappa_{\mathrm{r}}\left[\frac{\mathrm{d} T_{1}}{\mathrm{~d} r}\right]_{r_{0-}}=T_{1}^{0} \sqrt{\frac{\varrho c_{\mathrm{p}} \kappa_{\mathrm{r}}}{\pi t}}$.

Comparing this with the quantity $F_{\odot} \alpha_{0}$ in Eq. (53) we see that it can be neglected for times $t \gg t_{\text {ini }}$ with

$t_{\text {ini }}=\frac{\varrho c_{\mathrm{p}} \kappa_{\mathrm{r}}}{F_{\odot}^{2} \alpha_{0}^{2}}\left(T_{0}^{1}\right)^{2} \approx 20\left(T_{0}^{1}[\mathrm{~K}]\right)^{2} \mathrm{~s}$.

Although the cooling front in the radiation zone is timedependent, its influence on the temperature profile in the region $r>r_{0}$ can be neglected for $t \gg t_{\text {ini }}$, so that Eqs. (52) and (53) yield:

$a=\frac{F_{\odot} \alpha_{0}}{\tilde{\kappa}}$.

Since the undisturbed convection rapidly smoothes out the temperature perturbations for $r>r_{1}$, we can assume that $T_{1}\left(r_{1}, t\right)=0$ so that we obtain $T_{1}^{0}$ from Eq. (52):

$T_{1}^{0}=-\frac{F_{\odot} \alpha_{0}}{\tilde{\kappa}}\left(r_{1}-r_{0}\right)+\frac{1}{2} \frac{H_{\mathrm{rad}}}{\tilde{\kappa}}\left(r_{1}-r_{0}\right)^{2}$.

We see from Eq. (52) that the temperature perturbation grows outward in the interval

$r_{0} \leq r \leq r_{0}+\frac{F_{\odot} \alpha_{0}}{H_{\mathrm{rad}}}$
Solutions with larger values for $r$ are irrelevant since the temperature perturbation would then lead to $\delta>0$ in the upper part of the overshoot region, so that the magnetic field suppressing the convection could no longer be maintained in that layer. To avoid this situation, $r_{1}$ has to be in the range given by Eq. (58). For $r_{1}=r_{0}+F_{\odot} \alpha_{0} / H_{\text {rad }}$, the perturbations reach their peak values at $r=r_{0}$ :

$T_{1}\left(r_{0}\right)=-\frac{1}{2} \frac{F_{\odot}^{2} \alpha_{0}^{2}}{\tilde{\kappa} H_{\mathrm{rad}}}$

$\delta_{1} \simeq-\frac{H_{\mathrm{p}}}{T_{0}} \frac{\mathrm{d} T_{1}}{\mathrm{~d} r}=-\frac{H_{\mathrm{p}}}{T_{0}} \frac{F_{\odot} \alpha_{0}}{\tilde{\kappa}}$.

Using typical values for the solar overshoot region, $F_{\odot}=1.2 \times$ $10^{8} \mathrm{Wm}^{-2}, \alpha_{0}=0.4$, and $H_{\text {rad }}=3 \mathrm{Wm}^{-3}$ together with $\tilde{\kappa}=$ $10^{13} \mathrm{WK}^{-1} \mathrm{~m}^{-1}$ (comparable to the case $\epsilon=2$ on the left-hand side of Fig. 9), we obtain:

$r_{1}-r_{0}=16 \mathrm{Mm}$

$T_{1}\left(r_{0}\right)=-40 \mathrm{~K}$

$\delta_{1}\left(r_{0}\right)=-1.4 \times 10^{-4}$.

These values are in good agreement with the numerical results. From Eq. (55) we see that the influence of the radiative heat flux perturbation at the top of the radiation zone can be neglected for $t \gg 3 \times 10^{4} \mathrm{~s}$.

\section{Conclusions}

Radiative heating in regions of convergent radiative heat flux (Fan \& Fisher 1996; Moreno-Insertis et al. 2002) can have a significant effect on the storage of isolated magnetic flux tubes in the overshoot region of the Sun:

- The buoyancy resulting from radiative heating leads to a finite storage time. For a superadiabaticity of $\delta=-3 \times 10^{-6}$ (a typical value resulting from overshoot models based on the non-local mixing-length approach), the storage time is reduced to about a month.

- Storage of flux tubes for time scales comparable to the solar cycle requires rather strong subadiabaticity $\left(\delta \lesssim-10^{-4}\right)$.

- An equatorward flow within the overshoot region permits flux tube equilibria including the effects of radiative heating. However, for reasonable flow velocity of the order of $10 \mathrm{~m} \mathrm{~s}^{-1}$, such equilibria exist only for flux tubes with radius $\simeq 100 \mathrm{~km}$ and thus very little magnetic flux. The maximally storable flux in a single flux tube falls short of the flux of a typical sunspot $\left(\Phi \approx 10^{14} \mathrm{~Wb}\right)$ by about two orders of magnitude.

A magnetic field in the form of a magnetic layer, which reduces the convective flux and the convective heat conductivity in the whole overshoot region, leads to a stabilization of the stratification (more negative value of $\delta$ ), depending on the degree of the suppression:

- A reduction of the convective heat conductivity by a factor of $10^{2}$ leads to the rapid establishment of a new thermal equilibrium within a few months. The temperature in the overshoot region is reduced, leading to a stabilization of the stratification, so that values of $\delta<-10^{-4}$ can be reached. 
- A strong suppression by a factor $10^{4}$ leads to large temperature differences within the magnetized overshoot region. The upper part of the overshoot region is destabilized $(\delta>0)$, whereas the lowermost part becomes strongly subadiabatic. In this case, a magnetic field can be stably stored only in thin layer above the transition region towards the radiation zone.

We have thus shown that the magnetic field may improve the conditions for its own storage by reducing the convective transport in the overshoot region and thereby stabilizing the stratification. However, this works only as long as the magnetic field does not completely suppress the convective heat exchange. The remaining convective heat conductivity must be sufficiently large to prevent the formation of strong temperature perturbations $(\gtrsim 100 \mathrm{~K})$ in the overshoot region. If convection is suppressed completely, radiative heating destabilizes the stratification and leads to rapid flux loss except for a thin layer near the bottom of the overshoot region.

The change of the storage capability of the overshoot region with magnetic field strength may become important in view of the variation of $B$ in the course of the solar cycle. For low magnetic field strength, storage is facilitated owing to the increase of the subadiabaticity. Once the magnetic field is strong enough to suppress the convection nearly completely, the magnetic field becomes destabilized by the radiative heating, leading to an eruption of the stored flux.

This study shows that radiative heating in the overshoot region has a significant influence on the storage of magnetic flux. Superadiabaticities in the range $-10^{-6} \ldots-10^{-5}$, typical for non-local mixing-length models, are not sufficient for flux storage over time scales required for solar dynamo action. In this paper we discussed two possible solutions for this storage problem: 1 . The overshoot is more subadiabatic $\left(\delta<-10^{-4}\right)$ in the first place; 2 . A reduction of the vigor of the convection in the overshoot region by the magnetic field enlarges the subadiabaticity and thus enables flux storage.

A recent overshoot model (Xiong \& Deng 2001) and also numerical convection simulations favor larger subadiabaticities (see e.g. Brummell et al. 2002), which would completely solve the radiative heating problem. In the case of numerical overshoot simulations it is of course difficult to extrapolate results quantitatively to solar values, since the numerical parameters are orders of magnitudes away from the solar ones.

Our studies are based on a one dimensional approach describing the influence of the averaged convective quantities. A detailed description of the interaction between the convective motions and the stored field is not possible in this approach. Nevertheless the overall result that a suppression of convective motions in the overshoot stabilizes the stratification is not qualitatively affected by the details of the interaction between magnetic field and convection. Since the overshoot process results in an increase of the temperature gradient in the overshoot region (from the strongly subadiabatic radiative gradient close to the adiabatic gradient), any suppression of this process has to drive the system back to a higher subadiabaticity and thus stabilizes the stratification.
There is no doubt that future studies of the overshoot need to address the full nonlinear interaction between convection and magnetic field. But we have also to keep in mind that the contributions of numerical MHD-simulations to date need to be interpreted carefully, since they overestimate the influence of thermal conductivity (due to the restrictions on the achievable numerical resolution) and thus cannot describe the magnetic buoyancy correctly, which plays the crucial role in the storage problem. Also the coupling between the flow and the magnetic field may be overestimated due to the relative low magnetic Reynolds numbers achievable in numerical simulations.

Acknowledgements. I wish to thank M. Schüssler for essential discussions about the radiative heating and flux storage problem in the solar overshoot region. I am grateful to F. Moreno-Insertis and Y. Fan for helpful comments on a draft of this paper. This work has been funded by the Deutsche Forschungsgemeinschaft (DFG) under grant Schu 500-8.

\section{References}

Basu, S., Antia, H. M., \& Narasimha, D. 1994, MNRAS, 267, 209

Brummell, N. H., Clune, T. L., \& Toomre, J. 2002, ApJ, 570, 825

Caligari, P., Moreno-Insertis, F., \& Schüssler, M. 1995, ApJ, 441, 886

Caligari, P., Moreno-Insertis, F., \& Schüssler, M. 1998, ApJ, 502, 481

Choudhuri, A. R., \& Gilman, P. A. 1987, ApJ, 316, 788

Christensen-Dalsgaard, J., Monteiro, M. J. P. F. G., \& Thompson, M. J. 1995, MNRAS, 276, 283

Dorch, S. B. F., \& Nordlund, A.. 2001, A\&A, 365, 562

D'Silva, S., \& Choudhuri, A. R. 1993, A\&A, 272, 621

Duvall, T. L., \& Gizon, L. 2000, Sol. Phys., 192, 177

Fan, Y., \& Fisher, G. H. 1996, Sol. Phys., 166, 17

Fan, Y., Fisher, G. H., \& DeLuca, E. E. 1993, ApJ, 405, 390

Fan, Y., Fisher, G. H., \& McClymont, A. N. 1994, ApJ, 436, 907

Fisher, G. H., Fan, Y., Longcope, D. W., Linton, M. G., \& Pevtsov, A. A. 2000, Sol. Phys., 192, 119

Galloway, D. J., \& Weiss, N. O. 1981, ApJ, 243, 945

Moreno-Insertis, F. 1983, A\&A, 122, 241

Moreno-Insertis, F. 1986, A\&A, 166, 291

Moreno-Insertis, F. 1992, in NATO ASI Proc. 375: Sunspots. Theory and Observations (Dordrecht: Kluwer Academic Publishers), 385

Moreno-Insertis, F., Schüssler, M., \& Glampedakis, K. 2002, A\&A, 388,1022

Moreno-Insertis, F., Schüssler, M., \& Ferriz-Mas, A. 1992, A\&A, 264, 686

Parker, E. N. 1975, ApJ, 198, 205

Rekowski, B. V., \& Rüdiger, G. 1998, A\&A, 335, 679

Rempel, M., Schüssler, M., \& Tóth, G. 2000, A\&A, 363, 789

Schmitt, J. H. M. M., Rosner, R., \& Bohn, H. U. 1984, ApJ, 282, 316

Schüssler, M., Caligari, P., Ferriz-Mas, A., \& Moreno-Insertis, F. 1994, A\&A, 281, L69

Schüssler, M., \& Rempel, M. 2002, in From Solar Min to Max: Half a Solar Cycle with SOHO, ed. A. Wilson (ESTC, Noordwijk: ESA Publ. Div.), 499

Skaley, D., \& Stix, M. 1991, A\&A, 241, 227

Spruit, H. C. 1977a, Sol. Phys., 55, 3

Spruit, H. C. 1977b, Ph.D. Thesis

Tobias, S. M. 1996, ApJ, 467, 870

Tobias, S. M., Brummell, N. H., Clune, T. L., \& Toomre, J. 2001, ApJ, 549,1183

van Ballegooijen, A. A. 1982, A\&A, 113, 99

Xiong, D. R., \& Deng, L. 2001, MNRAS, 327, 1137 